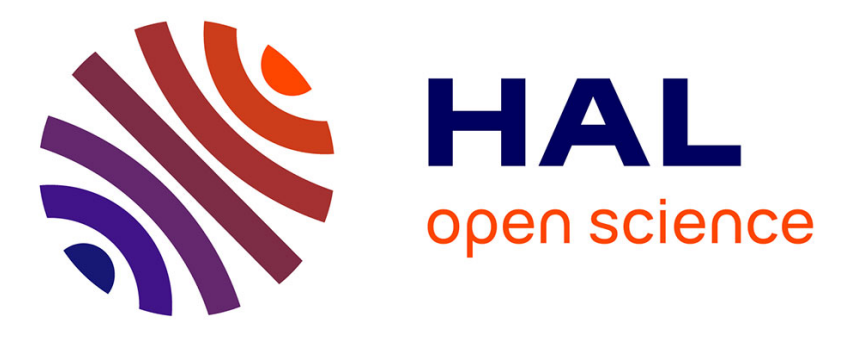

\title{
H 1 -Control of an ensemble of half-spin systems replacing Rabi pulses by adiabatic following
}

Ulisses Alves Maciel Neto, Paulo Sergio Pereira da Silva, Karine Beauchard, Pierre Rouchon

\section{- To cite this version:}

Ulisses Alves Maciel Neto, Paulo Sergio Pereira da Silva, Karine Beauchard, Pierre Rouchon. H 1 -Control of an ensemble of half-spin systems replacing Rabi pulses by adiabatic following. 58th Conference on Decision and Control - CDC 2019, Dec 2019, Nice, France. hal-02431019

\section{HAL Id: hal-02431019 \\ https://hal-mines-paristech.archives-ouvertes.fr/hal-02431019}

Submitted on 7 Jan 2020

HAL is a multi-disciplinary open access archive for the deposit and dissemination of scientific research documents, whether they are published or not. The documents may come from teaching and research institutions in France or abroad, or from public or private research centers.
L'archive ouverte pluridisciplinaire HAL, est destinée au dépôt et à la diffusion de documents scientifiques de niveau recherche, publiés ou non, émanant des établissements d'enseignement et de recherche français ou étrangers, des laboratoires publics ou privés. 


\title{
$H^{1}$-Control of an ensemble of half-spin systems replacing Rabi pulses by adiabatic following
}

\author{
Ulisses Alves Maciel Neto, Paulo Sergio Pereira da Silva ${ }^{\dagger}$ Karine Beauchard $\ddagger$ and Pierre Rouchon ${ }^{\S}$
}

\begin{abstract}
This work considers the control of an ensemble of non-interacting half-spin systems (Bloch equations) in a vertical static field $B_{0}$ subject to a pair of controlled radio-frequency inputs $\left(u_{1}(t), u_{2}(t)\right)$ acting on the horizontal plane. The state $M(t, \omega) \in S^{2}$ belongs to the Bloch sphere $S^{2}$, and it is indexed by the Larmor frequency $\omega \in\left(\omega_{*}, \omega^{*}\right)$. Previous works have constructed a local stabilizing feedback based on a Lyapunov functional which is essentially a convenient $H^{1}$-norm of a Sobolev space $H^{1}\left(\left(\omega_{*}, \omega^{*}\right), S^{2}\right)$ (see Beachard, Pereira da Silva and Rouchon [3]-[4]). This feedback assures local $L^{\infty}$-convergence of the initial state $M_{0}(\omega)$ to $-e_{3}$. However, the control law of that paper is a sum of a (infinite dimensional) state feedback with a T-periodic comb of $\pi$-Rabi pulses (Dirac impulses). The present work shows that one may replace this comb of Dirac pulses by adiabatic pulses. It is shown in the paper that, if $\left\|M_{0}(\omega)+e_{3}\right\|_{H^{1}}$ is small enough, our control strategy assures, for any $\varepsilon>0$, the existence of a pair $(T, \ell)$ where $T \in \mathbb{R}$ and $\ell \in \mathbb{N}$ such that $\| M(\ell T, \cdot)+$ $e_{3} \|_{L^{\infty}} \leq \varepsilon$. Simulations has shown that this new strategy produces faster convergence than the one that is based on the comb of Rabi pulses. The new method seems to work well even for initial conditions such that $\left\|M_{0}(\omega)+e_{3}\right\|_{H^{1}}$ is "relatively big".
\end{abstract}

${ }^{*}$ U. A. Maciel Neto is with Polythecnical School of University of So Paulo (USP), So Paulo, Brazil uma ciel@usp. br

${ }^{\dagger}$ P. S. Pereira da Silva is with Polythecnical School of University of So Paulo (USP), So Paulo, Brazil paulo@ lac.usp. br

${ }^{\ddagger} \mathrm{K}$. Beauchard is with Univ Rennes, CNRS, IRMAR - UMR 6625, F - 35000 Rennes, France karine.beauchard@ens-rennes.fr

$\S \mathrm{P}$. Rouchon is with Centre Automatique et Systèmes (CAS), Mines ParisTech, Paris, France pierre.rouchon@mines-paristech. fr

The first author was supported by CAPES (grant: ) and the second author was supported by FAPESP (grant: ) and CNPq (grant:

\section{INTRODUCTION}

Most controllability results available for infinite dimensional bilinear systems are related to systems with discrete spectra (see for instance, Beauchard and Coron, [1] for exact controllability results and Beauchard and Nersesyan, [5] Chambrion, Mason, Sigalotti, and Boscain, [7], Nersesyan [13], for approximate controllability results). As far as we know, very few controllability studies consider systems admitting a continuous part in their spectra. In Mirrahimi [12] an approximate controllability result is given for a system with mixed discrete/continuous spectrum: the Schrödinger partial differential equation of a quantum particle in an $\mathrm{N}$-dimensional decaying potential is shown to be approximately controllable (in infinite time) to the ground bounded state when the initial state is a linear superposition of bounded states. Ensemble controllability was studied for instance Li and Khaneja [10], [11] in the context of quantum systems that are described by Bloch equations depending continuously on a finite number of scalar parameters, and with a finite number of control inputs. The goal of ensemble controllability is to simultaneously steer a continuum of systems between states of interest with the same control inputs. Such continuous family of ordinary differential systems sharing the same control inputs can be seen as an interesting example of infinite dimensional systems with purely continuous spectra. The role of Lie algebras in the characterization of ensemble controllability was the main contribution of Li and Khaneja [11]. In Beauchard, Coron, and Rouchon [2], these aspects are studied under a functional analysis setting, developed for infinite dimensional systems governed by partial differential equations (see, e.g., Coron [8], for samples of these methods). Many results for this ensemble of Bloch equations, that include the discrimination between approximate and exact controllability are obtained in that paper. For instance, it is shown that a priori bounded $L^{2}$-controls are not sufficient to achieve exact controllability, but unbounded controls (containing a sum of Dirac masses) are able to recover controllability. In 
Beauchard et al. [3] it is shown that the ensemble of Bloch equations is approximately controllable to the south pole of the Bloch Sphere (in the Sobolev space $H^{1}$ ) in finite time. with unbounded controls.

In this paper one considers the ensemble $M(t, \omega) \in$ $S^{2} \subset \mathbb{R}^{3}$ of Bloch equations:

$$
\dot{M}(t, \omega)=S\left(u(t) e_{1}+v(t) e_{2}+\omega e_{3}\right) M(t, \omega),
$$

where $-\infty<\omega_{*}<\omega^{*}<+\infty, \omega \in\left(\omega_{*}, \omega^{*}\right)$, and $\left\{e_{1}, e_{2}, e_{3}\right\}$ is the canonical basis of $\mathbb{R}^{3}$, and $S(\cdot)$ is the (unique) antisymmetric matrix that defines the wedge product, that is, $S(c) v=c \wedge v$ for all $c, v \in \mathbb{R}^{3}$. Note that, for $c=c_{1} e_{1}+c_{2} e_{2}+c_{3} e_{3}$. one has:

$$
S(c)=\left[\begin{array}{ccc}
0 & -c_{3} & c_{2} \\
c_{3} & 0 & -c_{1} \\
-c_{2} & c_{1} & 0
\end{array}\right]
$$

It must be stressed that $u(t)$ and $v(t)$ are common controls for all the members of the ensemble, and they cannot depend on $\omega$. Such control problems have been addressed since a long time in the NMR community (see [16], [9]). For simplicity the derivative of $M$ with respect to time is denoted by $\dot{M}$, and the derivative of $M$ with respect to $\omega$ is denoted by $M^{\prime}$. To avoid confusion with derivation with respect to $\omega$, the transpose of a matrix $A$ will be denoted by $A^{\top}$. We shall consider only the Larmor dispersion (represented by the parameter $\omega$ ). One does not consider rf-inhomogeneity in this paper. We will state the control problem:

Definition 1 (Control Problem) Given an initial state $\left.M^{0} \in H^{1}\left(\left(\omega_{*}, \omega^{*}\right), S^{2}\right)\right)$, and given $\varepsilon>0$, choose $T_{f}>0$ and construct (bounded) controls $u:\left[0, T_{f}\right] \rightarrow \mathbb{R}$ and $v:\left[0, T_{f}\right] \rightarrow \mathbb{R}$ in a way that ${ }^{1}\left\|M\left(T_{f}, \cdot\right)+e_{3}\right\|_{L^{\infty}} \leq \varepsilon$

Beauchard et al [3] has investigated feedback stabilization of the ensemble (1), which possesses a continuous spectra. As in Mirrahimi (2009), the feedback design is based on a Lyapunov functional that is closely related to the norm of the state space $\left(H^{1}\right)$, a Banach space. This feedback assures local $L^{\infty}$-stabilization of the initial state $M_{0}(\omega)$ to $-e_{3}$. The control law that was considered in that paper is a sum of a bounded control $\left(u_{1}(t), u_{2}(t)\right)$ with a comb of $T$-periodic $\pi$-Rabi pulses.

The main contribution of the present work is to show that this comb of Rabi pulses may be replaced by an adiabatic following technique, which relies on a priori bounded $T$-periodic pair of inputs $(\bar{u}(t), \bar{v}(t))$.

The potential practical interest of such stabilization techniques consists in a simple algorithm providing an

\footnotetext{
${ }^{1}$ Recall that, for continuous functions, the $L^{\infty}$ norm is equivalen to the sup norm.
}

open loop control steering from the initial state to the final state. This algorithm just consists in the numerical integration of the closed loop system where the control values are recorded at each sample integration time.

The main results are presented in section 2. Computer simulations are presented in section 3. Those simulations have indicated a faster convergence than the original feedback law of [3] , [4]. The shape of the adiabatic pulses are presented in figure 6 (see appendix A). Some proof sketches are presented in the Appendix B.

\section{MAIN RESULTS AND HEURISTICS}

The main ingredients of our control strategy are the adiabatic following and the $H^{1}$-stabilizing strategy. One shall begin describing the adiabatic following and its (discontinuous) propagator.

\subsection{The adiabatic propagator}

Consider the adiabatic propagator equation:

$$
\dot{A}(t, \omega)=S\left(\bar{u}(t) e_{1}+\bar{v}(t) e_{2}+\omega e_{3}\right) A(t, \omega)
$$

where $A(t, \omega) \in S O(3)$, and $A(0, \omega)=I, \forall \omega \in\left[\omega_{*}, \omega^{*}\right]$ and the pair $(\bar{u}(t), \bar{v}(t))$ is the adiabatic control.

Consider that one applies the adiabatic control

$$
\begin{aligned}
\bar{u}(t) & =B_{1}(t) \sin \phi(t), \\
\bar{v}(t) & =B_{1}(t) \cos \phi(t),
\end{aligned}
$$

where $\phi(t)$ and $B_{1}(t)$ are defined by:

$$
\begin{aligned}
\dot{\phi}(t) & =\bar{k}(t) \bar{a}(t), \phi(0)=0 \\
B_{1}(t) & =\bar{k}(t) \bar{b}(t)
\end{aligned}
$$

where $\bar{a}(\cdot), \bar{b}(\cdot)$, and $\bar{k}(\cdot)$ are $T$-periodic functions defined by $\bar{a}(t)=a(t / T), \bar{b}(t)=b(t / T)$, and $\bar{k}(t)=$ $K k(t / T)$, where $a(\cdot), b(\cdot)$, and $k(\cdot)$ are 1-periodic normalized functions defined in the Appendix A, and $K>0$ is a chosen gain.

We shall re-initialize the propagator to the identity at $t_{0_{k}}=k T$, for $k \in \mathbb{N}$. One will denote the discontinuous $^{2}$ but $T$-periodic propagator $A: \mathbb{R} \times\left[\omega_{*}, \omega^{*}\right) \rightarrow$ $S O(3)$ by $A(t, \omega)$. The left-limit $\lim _{t \rightarrow T^{-}} A(t, \omega)$ is denoted by $A\left(T^{-}, \omega\right)$

The following convergence result can be proved by using standard adiabatic techniques [15] or by averaging methods [14]:

Theorem 1 Fix $K>\max \left\{\left|\omega_{*}\right|,\left|\omega^{*}\right|\right\}$ Then $\lim _{T \rightarrow \infty}\left\|A\left(T^{-}, \omega\right)-I\right\|_{L^{\infty}}=\lim _{T \rightarrow \infty} \max _{\omega \in\left[\omega_{*}, \omega^{*}\right]} \| A\left(T^{-}, \omega\right)-$ $I \|=0$.

\footnotetext{
${ }^{2}$ The discontinuities occur at $t=k T$ for $k \in \mathbb{N}$
} 
It must be stressed that the last theorem only holds because of the particular symmetries of the adiabatic pulses described in Appendix A (see Figure 6).

\subsection{The $H^{1}$ control law of the auxiliary system}

One forgets for the moment that $A(t, \omega)$ has some discontinuities and considers the following rotating coordinate change:

$$
N(t, \omega)=A^{\top}(t, \omega) M(t, \omega) .
$$

In each interval $[k T,(k+1) T)$ one may show that ${ }^{3}$ :

$$
\dot{N}(t, \omega)=S\left[A^{\top}(t, \omega)\left(u_{1}(t) e_{1}+u_{2}(t) e_{2}\right)\right] N(t, \omega)
$$

where $u(t)=u_{1}(t)+\bar{u}(t)$ and $v(t)=u_{2}(t)+\bar{v}(t)$ and $N(k T, \omega)=A^{\top}(k T, \omega) M(t, \omega)=M(t, \omega), k \in \mathbb{N}$. This construction would imply that $N(t, \omega)$ may have discontinuities at $t=k T, k \in \mathbb{N}$. In this paper one regards the continuous solution $N(t, \omega)$ of (5):

Remark 1 (Very important) The system (5) with input $\left(u_{1}(t), u_{2}(t)\right)$ and initial condition $N_{0}=M_{0}$ will be called by Auxiliary System. Its (continuous) solution will be denoted by $N(t, \omega)$. This implies that the discontinuous map $M_{1}(t, \omega)=A^{\top}(t, \omega) N(t, \omega)$ will not be the corresponding (continuous) solution of (1), with input $u(t)=u_{1}(t)+\bar{u}(t)$ and $v(t)=u_{2}(t)+\bar{v}(t)$ and initial condition $M_{0}$. In appendix $B$ it is shown that $M_{1}(t, \omega)=A^{\top}(t, \omega) N(t, \omega)$ is only an approximation of the (continuous) solution $M(t, \omega)$.

Consider the Lyapunov functional

$\mathscr{L}=\frac{1}{2}\left\|N+e_{3}\right\|_{H^{1}}^{2}=\int_{\omega_{*}}^{\omega^{*}}\left[\frac{1}{2}\left\langle N^{\prime}, N^{\prime}\right\rangle+1+\left\langle N, e_{3}\right\rangle\right] d \omega$

In order to compute $\dot{\mathscr{L}}$ note that $\xi=u_{1}(t) e_{1}+u_{2}(t) e_{2}$ does not depend on $\omega$. One has

$$
\dot{N}^{\prime}=S\left(A^{\top} \xi\right) N^{\prime}+S\left(\left(A^{\prime}\right)^{\top} \xi\right) N
$$

Hence

$$
\begin{aligned}
\dot{\mathscr{L}} & =\int_{\omega_{*}}^{\omega^{*}}\left\langle N^{\prime},\left[\left(A^{\top}\right)^{\prime} \xi \wedge N\right]\right\rangle+\left\langle e_{3},\left[\left(A^{\top}\right) \xi \wedge N\right]\right\rangle d \omega \\
& =H_{1} u_{1}+H_{2} u_{2}
\end{aligned}
$$

where

$$
H_{i}=\int_{\omega_{*}}^{\omega^{*}}\left\langle N^{\prime},\left[\left(A^{\top}\right)^{\prime} e_{i} \wedge N\right]\right\rangle+\left\langle e_{3},\left[\left(A^{\top}\right) e_{i} \wedge N\right]\right\rangle d \omega
$$

\footnotetext{
${ }^{3}$ Using the fact that $A S(\xi) v=S(A \xi) A v$ for all $\xi, v \in \mathbb{R}^{3}$ and $A \in$
}

for $i=1,2$. One may construct the control law

$$
u_{i}(t)=-H_{i}(A(t, \cdot), N(t, \cdot)), \quad i=1,2 .
$$

obtaining

$$
\dot{\mathscr{L}}=-\left(H_{1}^{2}+H_{2}^{2}\right)
$$

The closed loop system (5)-(6a)-(6b) with initial condition $N_{0}(\omega)=M_{0}(\omega)$ will be called the closed loop auxiliary system.

\subsection{Main result}

Before stating the main result, note that system (5) is parameterized by $T$ in the sense that the adiabatic inputs (4) depends on $T$, and so is the adiabatic propagator $A(t, \omega)$. This implies that the (continuous) solution $N(t, \omega)$ of (5) depends on the chosen $T$. The next theorem assures the solution of the problem of Def. 1.

Theorem 2 There exists $\delta>0$, such that for all $\varepsilon>0$ and any initial condition $M_{0} \in H^{1}\left(\left(\omega_{*}, \omega^{*}\right), \mathbb{S}^{2}\right)$ such that $\left\|M_{0}+e_{3}\right\|_{H^{1}}<\delta$, then there exist ${ }^{4} T>0$ and $\ell \in \mathbb{N}$ and a control law $\Omega:[0, \ell T] \rightarrow \mathbb{R}^{2}$ such that $\left\|M(\ell T, \cdot)+e_{3}\right\|_{L^{\infty}} \leq \varepsilon$. Furthermore, for the chosen $T$ and $\ell$, the control law $\Omega(t)$ to be applied to (1) can be obtained in the following way:

Step 1: Determine the adiabatic control $\bar{\Omega}(t)=(\bar{u}(t), \bar{v}(t))$ given in (3).

Step 2: Compute the solution of the closed loop system (5)-(6a)-(6b) with initial condition $N_{0}=M_{0}$ in the interval $[0, \ell T]$, obtaining the feedback law $\left(u_{1}(t), u_{2}(t)\right)$ given by $(6 a)-(6 b)$.

Step 3: Apply $\Omega(t)=(u(t), v(t))=\left(u_{1}(t)+\right.$ $\left.\bar{u}(t), u_{2}(t)+\bar{v}(t)\right)$ to the system $(1)$.

\section{COMPUTER SIMULATIONS}

One has chosen a priori $T=20, s_{0}=0.1, K=10$, $\ell=16$ and $T_{f}=\ell T$ for the new method ${ }^{5}$. As defined in (6a)-(6b), we have chosen unitary gains of the feedback law (we mean, there is no gain multiplying $H_{i}$ of $(6 \mathrm{~b})$ ). For the old method ${ }^{6}$, we have chosen $T=1$ and unitary gains as well. We have verified that greater values of $T$ than 1 for the old method are worse, but smaller values of $T$ will not improve the result. Figure 1 shows the simulation results in the Bloch sphere for these choices.

The obtained error of the adiabatic propagator is $\left\|A\left(T^{-}, \cdot\right)-I\right\| \leq 0.0009$ obtained in our computer simulation. So $\varepsilon_{2}=\ell\left\|A\left(T^{-}, \cdot\right)-I\right\| \leq 0.015$. It is very

\footnotetext{
${ }^{4}$ The proof of Theorem 2 shows that the values of $T$ and of $\ell$ must be big enough.

${ }^{5}$ See Appendix A for the definition of $s_{0}$.

${ }^{6}$ One has simulated the method of [4] considering the trivial target profile identically equal to $-e_{3}$. This is essentially equal to the method of [3].
} 
small in this case. In the simulations we have found that $\varepsilon_{1}=\left\|N(\ell T)+e_{3}\right\|_{L^{\infty}}$ is more than ten times greater than $\varepsilon_{2}$. Hence one will show only the behaviour of the auxiliary state $N(t, \omega)$.

In figure 1 one may see the initial condition $N_{0}=$ $M_{0}$ and the final condition $N(\ell T)$. One has obtained $\varepsilon_{2}=\left\|N\left(T_{f}\right)+e_{3}\right\|_{L^{\infty}}=0.185$ with our new method, and $\varepsilon_{2}=0.58$ with our old method. Both methods have considered the same unitary gains multiplying $H_{i}$ (see (6a)-(6b)). The expressions of the feedback of the old method is analogous to (6a)-(6b), with the difference that $A(t, \omega)$ is replaced by the matrix $\exp \left(S \omega e_{3} \sigma(t)\right)$, where $\dot{\sigma}(t)=(-1)^{E(t / T)}, E(s)$ is the integer part of $s$, and $\sigma(0)=0)$. Our new method have produced a result that is more than 3 times better than the old method with respect to the final $L^{\infty}$ norm..

Figure 2 regards only the new method. It shows the evolution of the Lyapunov functional $\mathscr{L}(t)=\frac{1}{2} \| N(t)+$ $e_{3} \|_{H^{1}}^{2}$. In that figure one shows also the evolution of $\left\|N(t)+e_{3}\right\|_{L^{\infty}}$ The controls $u_{1}(t)$ and $u_{2}(t)$ are also depicted in that figure. Figure 3 is a "zoom" of the last one. This allows to see the "microstructure" of the control of the new strategy.

Figure 4 shows the plot of $\log \left(\left\|N(t)+e_{3}\right\|_{H^{1}}^{2}\right)$ versus time. The inclination of the curves of $\log (\| N(t)+$ $\left.e_{3} \|_{H^{1}}^{2}\right)$ would give a measure of the exponential rate of decaying of $\left\|N(t)+e_{3}\right\|_{H^{1}}^{2}=2 \mathscr{L}(t)$. One sees that the convergence is not exponential, the inclination is decreasing as time passes for both methods. However, the inclination is much bigger for the first method in the beginning, and this inclination decreases faster for the old method with respect to the new one. This indicates that the new method seems to be more effective than the old one.

The Figure 5 presents a comparison of the input norms of the old and the new method.

\section{CONCLUSIONS AND FUTURE RE- SEARCH}

The main result of this work indicates that the Rabi pulses that are commonly encountered in Nuclear Magnetic Ressonance (NMR) techniques (for instance spinecho pulses) are not a mandatory ingredient for an efficient open loop control law. One might ask if this could imply that one may develop NMR methods with pulses with less intensity than the ones that are found in the present state of the art. This could be an interesting topic of future research, which may lead to produce less "agressive" NMR techniques for medical (and other possible) applications

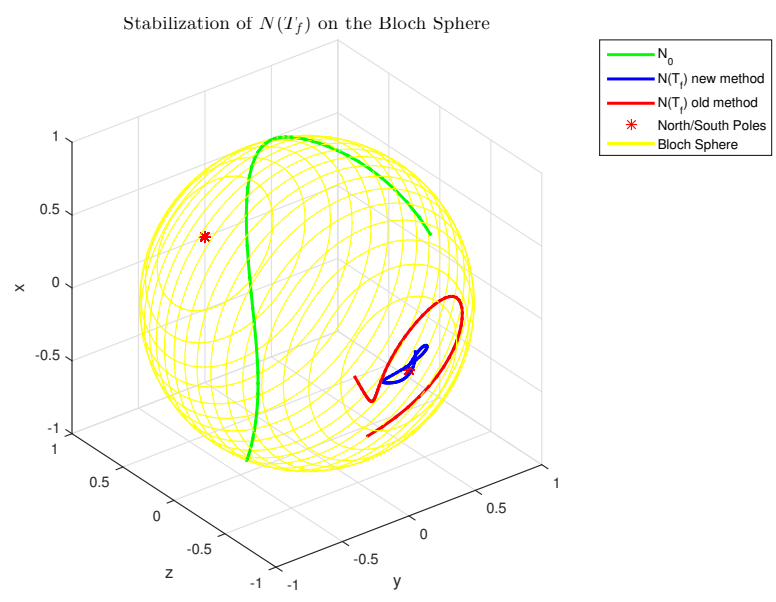

Figure 1. Results on the Bloch sphere for both the new and the old method.
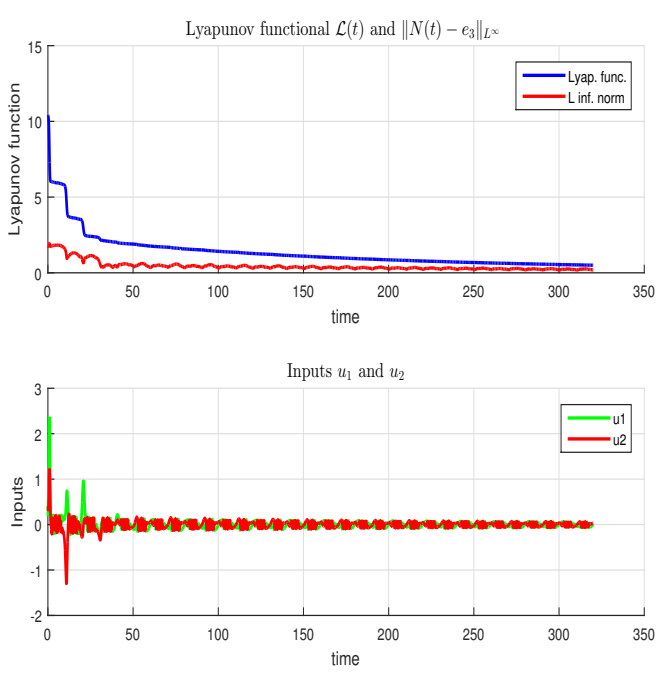

Figure 2. Lyapunov functional and inputs for the new method.

\section{ACKNOWLEDGMENTS}

The first author is supported by CAPES. The second author is supported by CNPq and FAPESP.

\section{A. Definition of normalized functions $a(s), b(s)$, and $k(s)$}

In this appendix we define the functions $s),(s)$, and $k(s)$ that are used in the adiabatic control. A computer simulation is presented in order to illustrate the convergence result (Theorem 1). For this, let $s_{0} \in(0,1 / 4)$. 

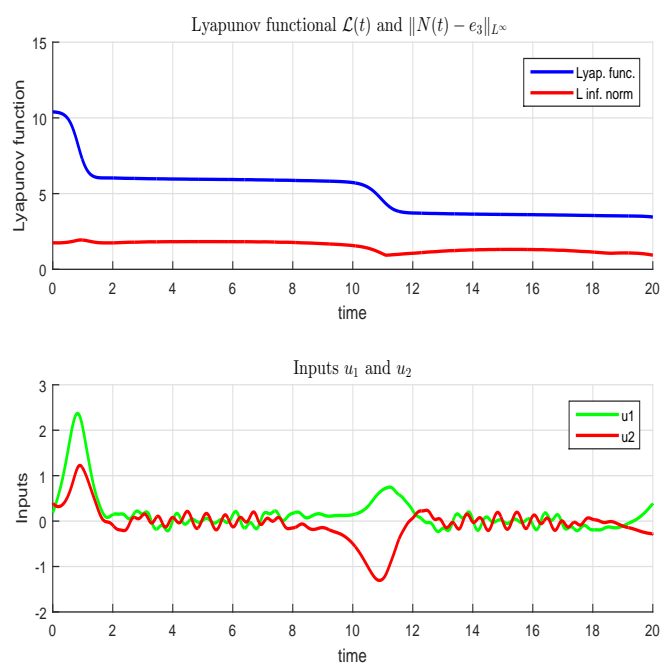

Figure 3. Lyapunov functional and inputs for the new method (zoom).

Define the function $a:[0,1] \rightarrow \mathbb{R}$ by (see Figure 6 ):

$$
a(s)=\left\{\begin{array}{l}
-1, \text { if } s \in\left[0, s_{0}\right] ; \\
-\cos \left[\frac{2 \pi\left(t-s_{0}\right)}{\left(1-4 s_{0}\right)}\right], \text { if } s \in\left(s_{0}, \frac{1}{2}-s_{0}\right] ; \\
1, \text { if } s \in\left(1 / 2-s_{0}, 1 / 2+s_{0}\right] ; \\
-\cos \left[\frac{2 \pi\left(t-3 s_{0}\right)}{\left(1-4 s_{0}\right)}\right], \text { if } s \in\left(\frac{1}{2}+s_{0}, 1-s_{0}\right] ; \\
-1, s \in\left(1-s_{0}, 1\right] .
\end{array}\right.
$$

Define the function $b(\cdot)$ by

$$
\bar{b}(s)=1-\{\bar{a}(s)\}^{2}
$$

and $k(\cdot)$ by

$$
\bar{k}(s)=\left\{\begin{array}{r}
1, \text { if } s \in[0,0.5), \\
-1, \text { if } s \in[0.5,1]
\end{array}\right.
$$

One may extend these functions $a, b, k$ to be 1 -periodic functions in a natural way. Figure 6 shows these functions for $s_{0}=0.1$.

A computer simulation of the adiabatic propagator $A(t, \omega)$ was done for $T=10, T=15$ and $T=20$, with $s_{0}=0.1$ and $K=10$. The values of $\left\|A\left(T^{-}, \omega\right)-I\right\|$ as a function of $\omega$ is given in Figure 7. The fast convergence of the maximum value of this norm to zero when $T \rightarrow \infty$ is easily seen in that figure.

\section{B. Proof Sketches}

\section{B.1. Auxiliary Results}

In order to prove the main result, one proves first the following important results

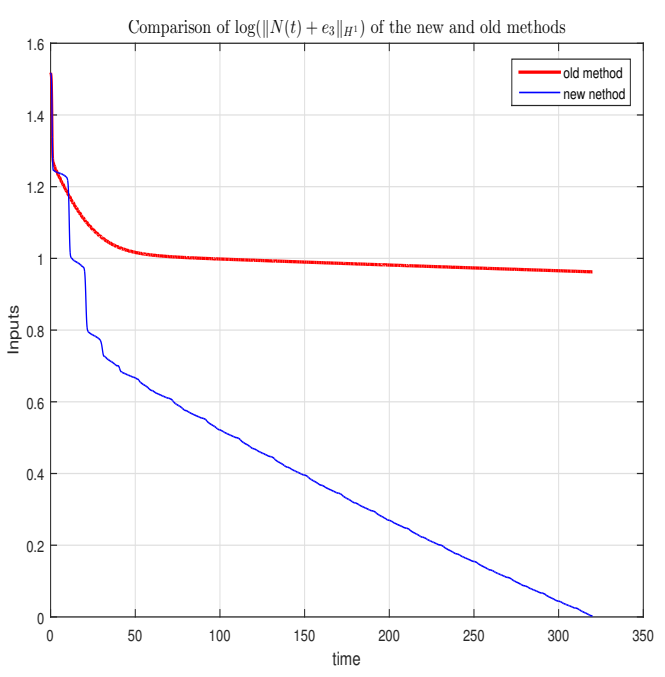

Figure 4. Plot of the natural logarithm of the square of the $H^{1}$ norm of $\left(N(t)+e_{3}\right)$.

Theorem 3 Fix $t_{0}=k T$ for some $k \in \mathbb{N}$. Let $^{7} T_{0}>0$ and $\tau_{0}=s_{0} T_{0}$ with $s_{0} \in(0,1 / 4)$. There exists $\delta>0$ with the following property: for all $\varepsilon>0$, there exist $c>0$ such that, for every $T \geq T_{0}$, and for every initial condition $N_{0}=N\left(t_{0}\right)$ such that $\left\|N_{0}+e_{3}\right\|_{H^{1}}<\delta$, and $\left\|N_{0}+e_{3}\right\|_{L^{\infty}} \geq \varepsilon$, then, one will have $\mathscr{L}\left(N\left(t_{0}+\tau_{0}\right)\right) \leq$ $\left(\mathscr{L}\left(N_{0}\right)-c\right)$ for system (5) in closed loop with the control law (6a)-(6b).

The proof of Theorem 3 is based on the following Lemmas, whose proofs may be found in [4] (particularized for $R=I$ ):

Lemma 1 Fix $t_{0}=k T$. There exists $\delta>0$ such that, if $\left\|N_{0}(\omega)+e_{3}\right\|<\delta$ and the control law $\left(u_{1}(t), u_{2}(t)\right)$ defined by $(6 a)-(6 b)$ is null on $\left[t_{0}, t_{0}+\tau_{0}\right]$, then $N_{0}=$ $-e_{3}$.

The following result is also similar. Since the auxiliary system is $T$-periodic, we shall state the result for $t_{0}=0$.

Lemma 2 Consider a sequence of initial conditions $\left(N_{0}^{n}\right)_{n \in \mathbb{N}}$ of $H^{1}\left(\left[\omega_{*}, \omega_{*}\right], S^{2}\right)$ such that $N_{0}^{n} \rightarrow N_{0}^{\infty}$ weakly in $H^{1}$ and the associated controls $\left(\Omega^{n}\right)_{n \in \mathbb{N}}, \Omega^{\infty}$. Then the solution $N^{n}(t, \cdot) \rightarrow N^{\infty}(t, \cdot)$ weakly in $H^{1}$ and $\Omega^{n}(t) \rightarrow \Omega^{\infty}(t)$ for $t \in\left[0, \tau_{0}\right]$.

Proof. (Of Theorem 3) Let $\gamma=\left\|N_{0}(\omega)+e_{3}\right\|_{H^{1}}<\delta$, where $\delta$ is defined in Lemma 1 . Since the auxiliary system is $T$-periodic, there is no loss of generality in considering $t_{0}=0$. The proof of this theorem is based on Lemmas 1 and 2. By contradiction, if the result does

\footnotetext{
${ }^{7}$ By construction, for $T \geq T_{0}$ the adiabatic control $(\bar{u}, \bar{v})$ is null for $\left.t \in\left[0, \tau_{0}\right]\right)$.
} 


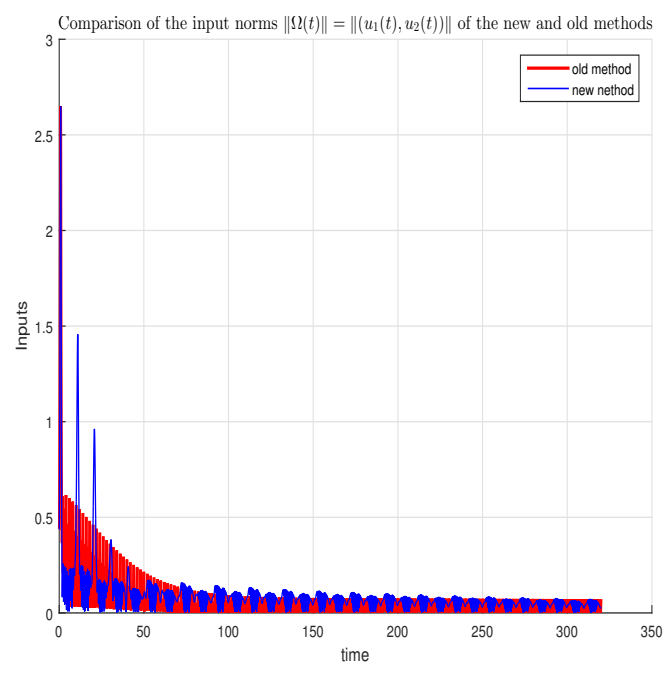

Figure 5. Plot of input norms $\left\|u_{1}(t)+u_{2}(t)\right\|$.

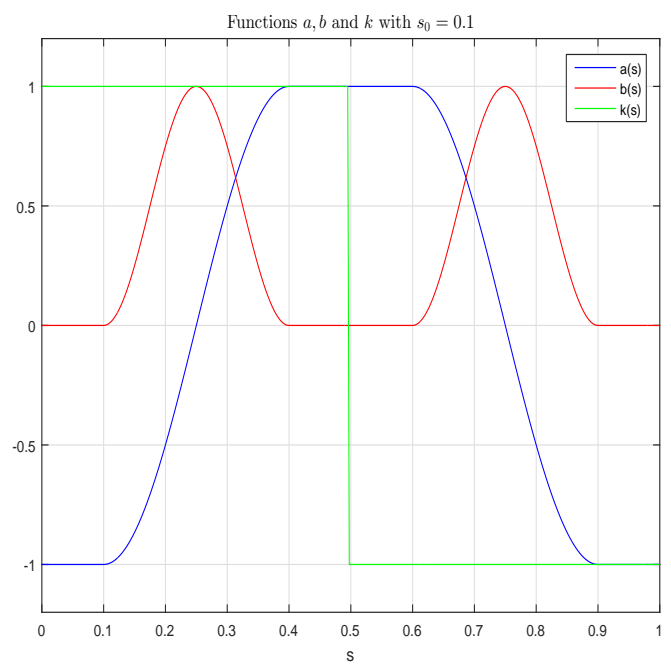

Figure 6. Functions $a, b$, and $k$ with $s_{0}=0.1$.

not hold, one may construct a sequence $N_{0}^{n}, n \in \mathbb{N}$ of initial conditions of the auxiliary system with the following properties:

(i) $\left\|N_{0}^{n}+e_{3}\right\|_{L^{\infty}} \geq \varepsilon, \forall n \in \mathbb{N}$;

(ii) $\left\|N_{0}^{n}+e_{3}\right\|_{H^{1}} \leq \gamma, \forall n \in \mathbb{N}$;

(iii) $\int_{0}^{\tau_{0}}\left[\left(u_{1}^{n}\right)^{2}(t)+\left(u_{2}^{n}\right)^{2}(t)\right] d t \leq 1 / n, \forall n \in \mathbb{N}, n>0$.

By (ii), passing to a convenient subsequence if necessary, one may assume $N_{0}^{n} \rightarrow N_{0}^{\infty}$ weakly in $H^{1}$. In particular, $N_{0}^{n} \rightarrow N_{0}^{\infty}$ strongly in the $L^{\infty}$ norm. Moreover, due to weak convergence, $\left\|N_{0}^{\infty}+e_{3}\right\|_{H^{1}} \leq \lim _{n \rightarrow \infty} \inf \| N_{0}^{n}+$ $e_{3} \|_{H^{1}} \leq \delta[6]$.

Now we shall show that the initial condition $N_{0}^{\infty}$ produces null controls for $t \in\left[0, \tau_{0}\right]$, which will be a contradiction with respect to Lemma 1 . Now, by the Lemma 2 , one has that $\Omega_{n}(t)=\left(u_{1}^{n}(t), u_{2}^{n}(t)\right) \rightarrow \Omega_{\infty}(t)$ where

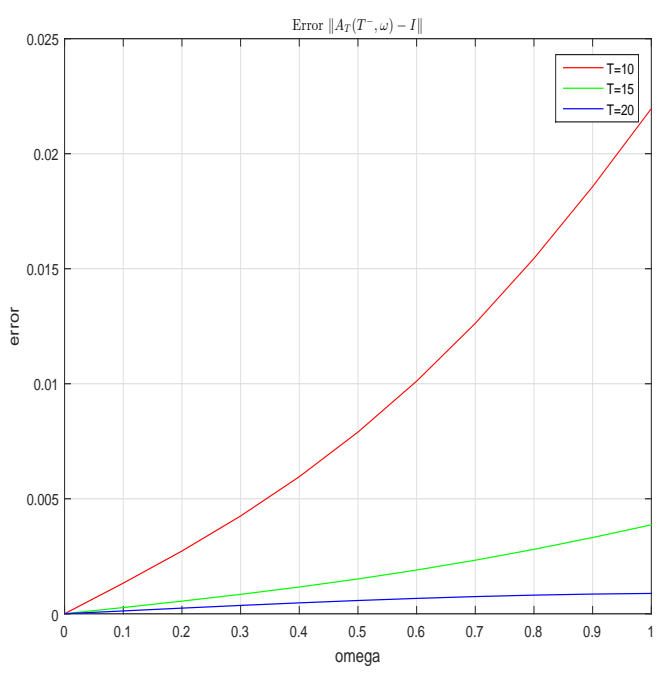

Figure 7. Plot of the Frobenius norm $\left\|A\left(T^{-}, \omega\right)-I\right\|$ as a function of $\omega$ with $s_{0}=0.1$ and $K=10$, for $T=10, T=15$ and $T=20$.

$\Omega_{\infty}(t)$ is the control that is obtained with the initial condition $N_{0}^{\infty}$. An extra work shows that the controls are of class $C^{1}$, and they are uniformly bounded, as well as their time-derivatives. In particular, the sequence of controls $\Omega_{n}(t)$ are uniformly bounded and equicontinuous, and so by Ascoli-Arzela theorem, passing to a subsequence if necessary, $\Omega_{n}$ converges to $\Omega_{\infty}$ in $C^{0}$ with the sup norm. Assuming that $\Omega_{\infty}$ is not identically null, this gives a contradiction with the fact that the $L^{2}$ norm of $\Omega_{n}$ tends to zero.

In order to prove the next results, one introduces the Propagator equation of the original system (1):

$$
\dot{B}(t, \omega)=S\left[u(t) e_{1}+v(t) e_{2}+\omega e_{3}\right] B(t, \omega)
$$

with initial condition $B_{0}(\omega)=B(0, \omega) \in$ $H^{1}\left(\left(\omega_{*}, \omega^{*}\right), S O(3)\right)$. By proposition 1 of [4], there exists $B_{0} \in H^{1}\left(\left(\omega_{*}, \omega^{*}\right), S O(3)\right)$ such that $B_{0} e_{3}=M_{0}$. It is then clear that the solution of the original system (1) is such that $M(t, \omega)=B(t, \omega) e_{3}$, where $B(t, \omega)$ is the solution of (9) and $M(t, \omega)$ is the solution of (1) with the same applied input $(u(t), v(t))$. It is also important to consider the propagator equation that is related to system (5) given by:

$$
\dot{C}(t, \omega)=S\left[A^{\top}(t, \omega)\left(u_{1}(t) e_{1}+u_{2}(t) e_{2}\right)\right] C(t, \omega)
$$

One denotes by $C(t, \omega)$ the (continuous) solution of (10) with the same initial condition $C_{0}=B_{0}$ and input $\left(u_{1}(t), u_{2}(t)\right)$ such that $u(t)=u_{1}(t)+\bar{u}(t)$ and $v(t)=$ $u_{2}(t)+\bar{v}(t)$. It is then clear that $N(t, \omega)=C(t, \omega) e_{3}$, 
where $N(t, \omega)$ is the solution of (5). Define the (discontinuous) map $B_{1}(t, \omega)=A(t, \omega) C(t, \omega)$, where $A(t, \omega)$ is the ( $T$-periodic) adiabatic propagator defined in (3). The following proposition regards the transformations between solutions of (1) and (5) and between solutions of (9) and (10):

Proposition 1 Assume that $M_{1}(t, \omega)=A(t, \omega) N(t, \omega)$. Then, the state $N(t, \omega)$ is a solution of (5) in the interval $[k T,(k+1) T)$ with input $\left(u_{1}(t), u_{2}(t)\right)$ if and only if the state $M_{1}(t, \omega)$ is a solution of (9) in the same interval with input $u(t)=u_{1}(t)+\bar{u}(t)$ and $v(t)=u_{2}(t)+\bar{v}(t)$. Similarly, assume that $B_{1}(t, \omega)=A(t, \omega) C(t, \omega)$. Then, the propagator $C(t, \omega)$ is a solution of $(10)$ in the interval $[k T,(k+1) T)$ with input $\left(u_{1}(t), u_{2}(t)\right)$ if and only if the propagator $B_{1}(t, \omega)$ is a solution of (9) in the same interval with input $u(t)=u_{1}(t)+\bar{u}(t)$ and $v(t)=u_{2}(t)+\bar{v}(t)$.

In one hand, if one fixes a (continuous) solution $B(t, \omega)$ of the propagator equation (9), one will obtain a "discontinuous solution" $C_{1}(t, \omega)=A_{T}^{\top}(t, \omega) B(t, \omega)$ of the propagator equation (10). On the other hand, we shall consider (continuous) solutions of the auxiliary system (10), that will produce a "discontinuous solution" $B_{1}(t, \omega)=A(t, \omega) C(t, \omega)$ :

Lemma 3 Consider the corresponding (continuous) solution of $C(t, \omega)$ of the propagator equation (10) that is obtained by the application of an input $\left(u_{1}(t), u_{2}(t)\right)$ in an interval $\left[0, T_{f}\right]$ with initial condition $C_{0}=$ $B_{0}$. Let $B_{1}(\cdot, \omega)$ be the map defined by $B_{1}(t, \omega)=$ $A(t, \omega) C(t, \omega)$, where $A(t, \omega)$ is the $T$-periodic map defined in section 2.1. Then the (continuous) solution $B(t, \omega)$ of (9) that is obtained with with the same initial condition $B_{0}=C_{0}$ and applying the input $(u(t), v(t))=\left(u_{1}(t)+\bar{u}(t), v_{1}(t)+\bar{v}(t)\right)$ is such that $\left\|B(\ell T, \omega)-B_{1}(\ell T, \omega)\right\| \leq \ell\|A(T, \omega)-I\|$.

Proof. See appendix B.2.

\section{B.2. Proof of Lemma 3}

The proof of the Lemma 3 is a consequence of the right-invariance of the propagator equation and the fact that the Frobenius norm is invariant by right- and leftmultiplications by orthogonal matrices.

Proposition 2 Fix $\omega \in\left(\omega_{*}, \omega^{*}\right)$. The following affirmations are equivalent for the propagator equation (9):

- $B_{1}(t, \omega)$ and $B(t, \omega)$ are solutions of $(9)$ in the interval $\left[t_{0}, t_{1}\right)$ with the same applied inputs $u(t)$ and $v(t)$.
- There exists a right-translation $R(\omega) \in S O(3)$ such that $B(t, \omega)=B_{1}(t, \omega) R(\omega)$. Furthermore, the Frobenius norm $\left\|B_{1}(t, \omega)-B(t, \omega)\right\|$ is constant in $\left[t_{0}, t_{1}\right)$ and given by $\|R(\omega)-I\|$.

The proof of the last proposition is a straightforward consequence of the right-invariance of (9), the uniqueness of solutions of (9) and the invariance of the Frobenius norm with respect of right- and left-multiplications by matrices in $S O(3)$. An easy consequence of the last proposition is the following one, which means that, if $B(\cdot, \omega)$ is a "continuous solution" of $(9)$ and $B_{1}(\cdot, \omega)$ is a "discontinuous solution" of (9), both with the same initial condition $B_{0}(\omega)=B_{1}(0, \omega)=B(0, \omega)$, then the Frobenius distance between $B(\cdot, \omega)$ and $B_{1}(\cdot, \omega)$ is bounded by the sum of discontinuities of $B_{1}$.

Proposition 3 Let $T_{f}=\ell T$ for some $\ell \in \mathbb{N}$. Assume that $B_{1}(\cdot, \omega):\left[0, T_{f}\right] \rightarrow$ is a map such that $B_{1}(t, \omega)$ is a solution of (9) in each interval $[k T,(k+1) T), k \in \mathbb{N}$ and $B(\cdot, \omega):\left[0, T_{f}\right] \rightarrow$ is another map with the same property. Assume that the map $B_{1}(\cdot, \omega)$ is continuous in each interval $[k T,(k+1) T)$ with possible discontinuities at $t=k T, k=1,2, \ldots, \ell$. Assume that the map $B(\cdot, \omega)$ is continuous in $\left[0, T_{f}\right]$. Assume that $B(0, \omega)=$ $B_{1}(0, \omega)=B_{0}(\omega)$. Then $\left\|B_{1}(t, \omega)-B(t, \omega)\right\| \leq$ $\sum_{k=1}^{\ell}\left\|B_{1}\left(k T^{-}, \omega\right)-B(k T+, \omega)\right\|, \forall t \in\left[0, T_{f}\right]$.

Now note that a (continuous) solution $C(t, \omega)$ of (10) is transformed into a discontinuous map $B_{1}(t, \omega)=$ $A(\omega, t) C(t, \omega)$. From Proposition $1, B_{1}(t, \omega)$ is a solution of the propagator equation (9) inside the intervals $[k t,(k+1) T)$. The "discontinuous" solution $B_{1}(t, \omega)$ is such that, at each instant $t=k T$ one has a discontinuity of $B_{1}$ of a distance $\| B_{1}\left(k T^{+}\right)-$ $B_{1}\left(k T^{-}\right)\|=\| A\left(k T^{+}\right) C\left(k T^{+}\right)-A\left(k T^{-}\right) C\left(k T^{-}\right) \|$. By the continuity of the map $C(\cdot, \omega)$, then $C=C\left(k T^{+}\right)=$ $C\left(k T^{-}\right)$. As the Frobenius distance is invariant by right- and left-multiplications by orthogonal matrices, then $\left\|A\left(k T^{+}\right) C-A\left(k T^{-}\right) C\right\|=\left\|A\left(k T^{+}\right)-A\left(k T^{-}\right)\right\|=$ $\left\|A\left(T^{-}, \omega\right)-I\right\|$ (recall that $\left.A\left(k T^{+}\right)=I\right)$. So, after $\ell$ periods of $T$ seconds, Proposition 3 implies that the Frobenius distance $\left\|B_{1}(\ell T, \omega)-B(\ell T, \omega)\right\|$ is not greater than the sum of all individual discontinuities. This shows Lemma 3.

\section{B.3. Proof of Theorem 2}

Proposition 4 Given $\varepsilon>0$ and $T_{0}>0$ there exist some $\delta>0$ and $\ell \in \mathbb{N}$ such that, if $\left\|M_{0}+e_{3}\right\|_{H^{1}}<\delta$, then $\left\|N(\ell T, \cdot)+e_{3}\right\|_{L^{\infty}} \leq \varepsilon$ for all $T>T_{0}$.

Proof. Let $c>0$ (that depends on $\varepsilon$ ) be the constant that is defined in Theorem 3. Let $p \in \mathbb{N}$ such 
that $\mathscr{L}\left(N_{0}\right)-p c \leq 0$. By contradiction, assume that $\left\|N(\ell T, \cdot)+e_{3}\right\|_{L^{\infty}} \geq \varepsilon$ for all $\ell \in\{0,1, \ldots, p\}$. Since the Lyapunov functional $\mathscr{L}(t)$ is nonincreasing, the repetitive application of Theorem 3 at the instants $t=k T$ for $k=0,1, \ldots, p$ would give $\mathscr{L}(N(p T)) \leq \mathscr{L}\left(N_{0}\right)-$ $p c<0$. This is not possible since the Lyapunov functional is always nonnegative. So there must exist some $\ell \in\{0,1, \ldots, p\}$ with the claimed property.

Proposition 5 Fixed $k \in \mathbb{N}$, the solution $M(t, \omega)$ of (1) with initial condition $M_{0}(\omega)$ is such that

$\|M(k T, \cdot)\|_{L^{\infty}} \leq k\left\|A\left(k T^{-}, \cdot\right)-I\right\|_{L^{\infty}}+\left\|N(k T, \cdot)+e_{3}\right\|_{L^{\infty}}$

Proof. Consider the original system (1) with initial condition $M_{0}$. Let $N(t, \omega)$ be the (continuous) solution of (5) with initial condition $N_{0}=M_{0}$. It is clear that

$$
M_{1}(t, \omega)=A(t, \omega) N(t, \omega)
$$

is a "discontinuous" solution of (1). Note that $M_{1}(t, \omega)=A(t, \omega) N(t, \omega)=A(t, \omega) C(t, \omega) e_{3}=$ $B_{1}(t, \omega) e_{3}$. Recall that the "continuous solution" $M(t, \omega)$ of the system (1) with initial condition $M_{0}=N_{0}$ is such that $M(t, \omega)=B(t, \omega) e_{3}$. Since $A(k T, \omega)=I$, then from Lemma 3 one gets $\left\|M(k T, \omega)+e_{3}\right\|=\| M-$ $M_{1}+M_{1}+e_{3}\|\leq\|\left(B-B_{1}\right) e_{3}\|+\| N(k T, \omega)+e_{3} \| \leq$ $k\left\|A\left(T^{-}, \omega\right)-I\right\|+\left\|N(k T, \omega)+e_{3}\right\|$ which completes the proof.

Proof. (of the Theorem 2) Fix $\varepsilon>0$ and choose $\varepsilon_{1}>0$ and $\varepsilon_{2}>0$ such that $\varepsilon=\varepsilon_{1}+\varepsilon_{2}$. It is possible to find $\ell \in \mathbb{N}, T_{0}>0$ and a control law $\Omega_{T}:\left[0, T_{f}\right] \rightarrow \mathbb{R}^{2}$ (depending on $T$ ), with $T_{f}=\ell T$, in a way that the application of $\Omega_{T}(t)=\left(u_{1}(t), u_{2}(t)\right)$ to system (5) furnishes $\left\|N(\ell, \cdot)+e_{3}\right\|_{L^{\infty}} \leq \varepsilon_{1}$ for all $T>T_{0}$.

Find $T^{\star}>T_{0}$ big enough (depending on $\ell$ ) such that $\ell\left\|A\left(T^{-}, \cdot\right)-I\right\|_{L^{\infty}} \leq \varepsilon_{2}$ and apply the open loop control $(u(t), v(t))=\Omega_{T^{\star}}(t)+(\bar{u}(t), \bar{v}(t))$ to system (1), obtaining $\left\|M(\ell, \cdot)+e_{3}\right\| \leq \varepsilon_{1}+\varepsilon_{2}=\varepsilon$.

\section{References}

[1] K. Beauchard and J.-M. Coron. Controllability of a quantum particle in a moving potential well. Journal of Functional Analysis, 232(2):328-389, 2006.

[2] K. Beauchard, J.-M. Coron, and P. Rouchon. Controllability issues for continuous spectrum systems and ensemble controllability of bloch equations. Communications in Mathematical Physics, 95(4):525-557, 2010.
[3] K. Beauchard, P. S. Pereira da Silva, and P. Rouchon. Stabilization for an ensemble of half-spin systems. Automatica, 48(1):68-76, 2012.

[4] K. Beauchard, P. S. Pereira da Silva, and P. Rouchon. Stabilization of an arbitrary profile for an ensemble of half-spin systems. Automatica, 49(7):2133-2137, 2013.

[5] K. Beauchard and V. Nersesyan. Semi-gobal weak stabilization of bilinear schrdinger equations. Comptes Rendus Mathématiques, 348(19):1073-1078, 2010.

[6] H. Brezis. Functional Analysis, Sobolev Spaces and Partial Differential Equations. Universitext. Springer, Piscataway, 2010.

[7] T. Chambrion, P. Mason, M. Sigalotti, and M. Boscain. Controllability of the discrete-spectrum schrödinger equation driven by an external field. Annales de lInstitut Henri Poincaré Analyse Non Linéaire, 26(1):329-349, 2009.

[8] J.-M. Coron. Control and Nonlinearity. American Mathematical Society, 2007.

[9] K. Kobzar, B. Luy, N. Khaneja, and S. J. Glaser. Pattern pulses: design of arbitrary excitation profiles as a function of pulse amplitude and offset. Journal of Magnetic Resonance, 173(2):229-235, 2005.

[10] J. S. Li and N. Khaneja. Control of inhomogeneous quantum ensemble. Physical Review A, 73:030302-, 2006.

[11] J. S. Li and N. Khaneja. Control of inhomogeneous quantum ensemble. IEEE Transactions on Automatic Control, 54(3):528-536, 2009.

[12] M. Mirrahimi. Lyapunov control of a quantum particle in a decaying potential. Annales de linstitut Henri Poincaré Analyse Non Linéaire, 26:1743-1765, 2009.

[13] V. Nersesyan. Growth of sobolev norms and controllability of schrödinger equations. Communications in Mathematical Physics, 290(1):371-387, 2009.

[14] U. A. Maciel Neto and P. S. Pereira da Silva. Controle adiabático de ensembles quânticos via método das médias. In Anais do XX Congresso Brasileiro de Automática, pages 2629-2636, September 2014.

[15] S. Teufel. Adiabatic Perturbation Theory in Quantum Dynamics. Lecture Notes in Mathematics. SpringerVerlag Berlin Heidelberg, 2003.

[16] R. Tycko. Broadband population inversion. Physical Review Letters, 51:775-777, 1983. 